\title{
Development of a patient-centred model of community- based OPAT (CoPAT) service in Singapore
}

\author{
$\mathrm{Xu} \mathrm{Yi}^{1 *}$, Lim Su Fee ${ }^{2}$, Fazila Aloweni ${ }^{3}$, Chua Ying Ying ${ }^{4}$ and Huang Jie Ying ${ }^{5}$ \\ ${ }^{1}$ Senior Nurse Clinician (Community Nurse), RHS-Community Nursing, Singapore General Hospital, Singapore \\ ${ }^{2}$ Assistant Director of Nursing (Advanced Practice Nurse), RHS-Community Nursing, Singapore General Hospital, Singapore \\ ${ }^{3}$ Nurse Clinician, Nursing Division, Singapore General Hospital, Singapore \\ ${ }^{4}$ Consultant, Department of Infectious Diseases, Singapore General Hospital, Singapore \\ ${ }^{5}$ Senior Staff Nurse, Department of Infectious Diseases, Singapore General Hospital, Singapore
}

\begin{abstract}
There is a growing trend of expanding Outpatient Parenteral Antibiotics Therapy (OPAT) services to the community as it is more cost-effective for both patients and healthcare organisations. At the Singapore General Hospital (SGH), frail patients requiring intravenous (IV) antibiotics often have to prolong their hospitalisation to complete the course of treatment.

Community OPAT (CoPAT) service is a joint effort between SGH Community Nursing and Department of Infectious Diseases. With the new CoPAT service, patients have the options of receiving their IV antibiotics at home rather than receiving treatment at the hospital or outpatient clinic.

This case study described the process of providing CoPAT services in the community, its challenges and learning points and the positive outcomes of cost saving and improved patient experience. Early preliminary screening of suitable patients and good communication link among hospitals and community teams are essential in ensuring its success.
\end{abstract}

\section{Introduction}

Outpatient Parenteral Antibiotic Therapy (OPAT) refers to the approach for delivering intravenous (IV) antimicrobials in the outpatient setting or community [1]. OPAT service was first described in the United States in 1974 and was subsequently introduced to Singapore in 2002 [2]. This service requires a multidisciplinary team with expertise and experience in antimicrobial therapy for safety and long-term sustainability [3]. It is further recommended that the OPAT team provides an extension of services including laboratory testing, catheter care, regular nursing and physician assessments, follow-up, and adjustments as part of the optimal care to the existing primary illness, comorbidities and emerging complications [2].

Globally, there is a growing trend of expanding OPAT services to the community as it is cost-effective, and it also provides greater comfort to the patient when he/she receives the IV antibiotic at home [4]. OPAT improves patient's satisfaction as it provides him/her the advantages of being able to resume his/her daily activities and having greater freedom and control at a familiar environment [5]. In additon, administering IV antibiotic at home reduces the risk of nosocomial complications as a result of prolonged hospitalisation [4].

In Singapore, OPAT services are mainly provided at specific outpatient clinics within acute hospitals. This service may not be accessible to patients who are frail or with limited mobility as it requires them to travel daily to the outpatient clinic and also incurs transportation costs. As a result, many of them would prefer to remain in the hospital till they receive the full course of IV antibiotics.
This paper aims to describe the development of a patient-centred model of community-based OPAT (CoPAT) service in Singapore using a case study approach.

\section{Background}

Community Nursing programme (funded by the Ministry of Health, Singapore) is envisaged as a key anchor for population health management and an enabler for person-centred care beyond hospitals' walls, into the community. Singapore General Hospital (SGH) Community Nursing Programme was officially launched on 28 February 2018 as part of the Regional Health System (RHS)'s ongoing efforts to strengthen community care across the five Communities of Care (CoC) Zones, i.e. Bukit Merah, Chinatown, Telok Blangah, Tiong Bahru and Katong (Figure 1). Communtiy Nurses aim to bridge the gap between care in the hospital and community and complement the services provided by the hospital, primary care sector and community service providers.

One of the challenges faced by the acute care hospital was discharging frail patients who required prolonged IV antibiotics. These patients were unable to utilise the OPAT services in the hospital due to their limited mobility. Hence, the CoPAT service was developed in SGH

${ }^{\star}$ Correspondence to: $\mathrm{Xu}$ Yi, Senior Nurse Clinician (Community Nurse), RHS Community Nursing, Singapore General Hospital, Singapore, E-mail: xu.yi@ sgh.com.sg

Received: August 27, 2019; Accepted: September 09, 2019; Published: September 12, 2019 


\section{SingHealth RHS(South-East) -}

\section{Community of Care Zones Concept}

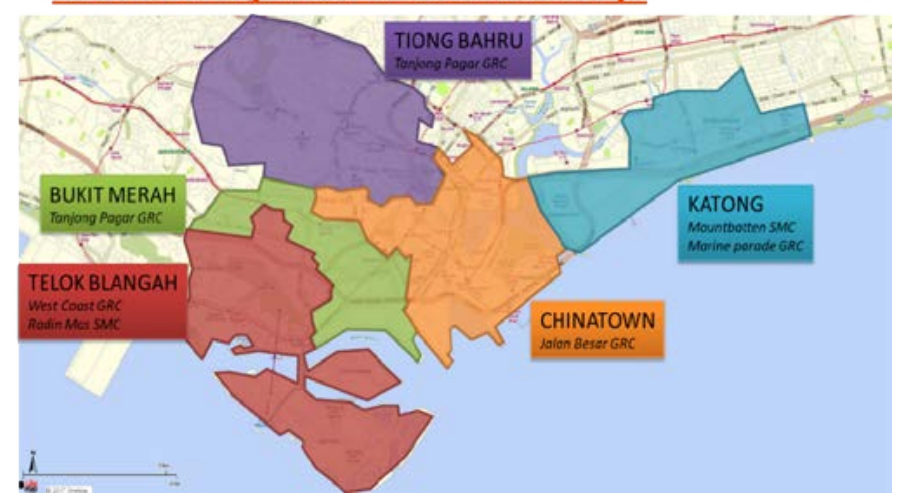

Figure 1. SingHealth RHS (South-East) 5 CoC Zones

on September 2018 after much deliberation on the risks and benefits, capabilities and service demand. The CoPAT service is a collaborative effort of various teams including OPAT, Division of Internal Medicine (DIM), Community Nursing, Pharmacy and Finance. CoPAT provides patients with an alternative to inpatient hospitalisation stay, thus reduces the associated risk of healthcare-associated infection due to prolonged hospitalisation. Careful attention was given to the development of clinical guidelines and workflow which guide the team's roles and responsibilities, practice standards and escalation protocols in ensuring risks are minimised and managed.

\section{Enrolment}

Patients are screened for their eligibility to enrol into the CoPAT programme. The inclusion criteria comprised the followings:

i. Require less than two weeks of IV Ceftriaxone and/or IV Meropenem; and

ii. Clinical Frailty Scale (CFS) of 4 and above (vulnerable to very severely frail) [6]; and

iii. certified fit for CoPAT by attending physician; and

iv. able to comprehend instructions; and

v. residing within SGH CoCs; and

vi. not discharged from the hospital against the doctor's advice.

\section{Patients are excluded from the programme if they have:}

i. poor venous access and/or has signs of surrounding skin and soft tissue infection; and/or

ii. history of unauthorized IV drug use.

The first dose of antibiotic is given during the patient's hospitalisation stay. This is to detect and manage complications early, if any. The following is a case study that illustrates the CoPAT service and some of the key challenges and learning points.

\section{Case report}

Madam G is a 99-year-old elderly who has no significant medical history. She was admitted for fever, associated with generalized malaise and lethargy. She was subsequently diagnosed with Cholangitis and her condition was further complicated by Klebsiella pneumonia and bacteraemia in which she required fourteen days of IV Ceftriaxone $2 \mathrm{~g}$ daily. She was previously community ambulant with a CFS score of 3 . After multiple episodes of frequent falls, she was deconditioned and her CFS score deteriorated to 5 . She is widowed and stays with her children and a domestic helper.

Under the care of the DIM and infection control teams, Madam $\mathrm{G}$ was referred for CoPAT. The OPAT team reviewed and assessed her eligibility for the programme. As this was a new service, the OPAT nurse made an appointment with Madam $G$ and her daughter to brief on the programme details, i.e. estimated charges for medication and consumables, vascular access, potential drug-specific complications or adverse effects and home visits by a community nurse.

Once enrolled in the CoPAT programme, the OPAT nurse communicated with the inpatient and community nursing teams on Mdm G's discharge plan and home visit schedules. Preparation for CoPAT such as prescriptions for IV medications, CoPAT kits, outpatient appointments and laboratory tests were arranged. The CoPAT kits were packed by the hospital's pharmacy staff based on a standard list of consumables for daily infusion (Set A) and IV cannulation (Set B). This process reduced the time spent by the ward nurse and pharmacy staff in sourcing the various pieces of items. Before discharge, the community nurse visited the patient in the hospital to conduct a baseline assessment and to establish rapport to allay any uncertainty about the treatment at home.

During the initial home visit, the community nurse used a standardised health assessment guide to examine Madam G, i.e. physical assessment, checking of vital signs, assessing the peripheral cannulation site for phlebitis and patency before the administration of IV medication. Once ensuring that Mdm G was fit to receive the IV Ceftriaxone, the nurse proceeded to verify and reconstitute the antibiotics (Figure 2). During infusion, Madam G was closely monitored for any complications. The nurse also took the opportunity to interact with Madam G, assessed her fall risks, recommended home safety measures and provided education on safety awareness.

IV Ceftriaxone was successfully administered to Mdm G daily at her home over a consecutive period of six days with no adverse effects. Madam G's family was satisfied with the home service as it managed to shorten her hospitalisation stay and at the same time, allowed her to resume her usual activities. The family also reported that they saw a significant improvement in her health state and her mood compared to the time when she was hospitalised.

\section{Discussion}

An early preliminary screening to determine whether the patient is a potential candidate for OPAT is vital as this service requires sufficient time to obtain patient's acceptance, provide education, assess IV access and check home nursing availability [3]. It could be challenging in

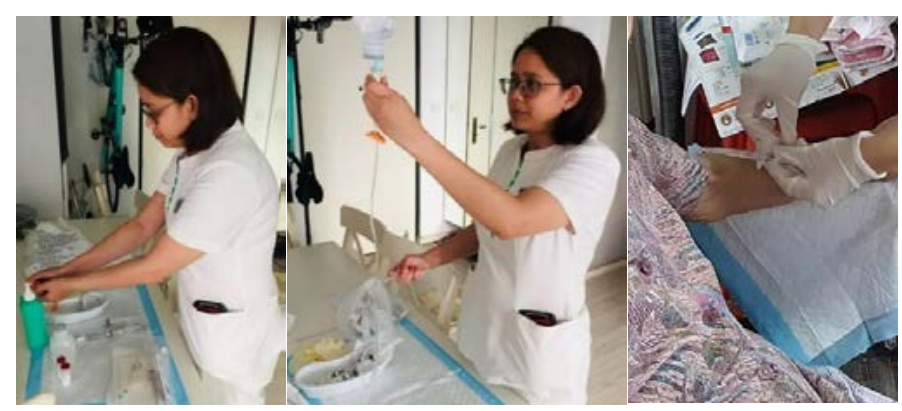

Figure 2. Nurse prepares and administrates IV antibiotics at home 
Table 1. Summary of CoPAT pilot cases

\begin{tabular}{|c|c|c|c|c|c|c|c|c|}
\hline $\mathbf{S} / \mathrm{N}$ & Name & Ward Class & LOS & $\begin{array}{l}\text { Bill Size (Before } \\
\text { Subsidy) }\end{array}$ & $\begin{array}{l}\text { Bill Size (After } \\
\text { Subsidy) }\end{array}$ & $\begin{array}{c}\text { Average Daily Bill } \\
\text { (After Subsidy) }\end{array}$ & Days of CoPAT & $\begin{array}{c}\text { Total Cost Saved } \\
\text { by Patient (After } \\
\text { Subsidy) }\end{array}$ \\
\hline 1 & Patient 1 & B2 & 6 & $\$ 7,950.62$ & $\$ 3,076.09$ & $\$ 513$ & 9 & $\$ 4,617$ \\
\hline 2 & Patient 2 & $\mathrm{C}$ & 7 & $\$ 6,802.17$ & $\$ 2,329.61$ & $\$ 333$ & 7 & $\$ 2,331$ \\
\hline 3 & Patient 3 & B2 & 7 & $\$ 13,748.49$ & $\$ 5,507.30$ & $\$ 787$ & 8 & $\$ 6,296$ \\
\hline \multirow[t]{2}{*}{4} & Patient 4 & B2 & 7 & $\$ 10,667.26$ & $\$ 3,975.16$ & $\$ 568$ & 6 & $\$ 3,408$ \\
\hline & & & & & & Grand Total & 30 & $\$ 16,652$ \\
\hline
\end{tabular}

identifying the suitable candidates as the CoPAT service was new to the ground staff. Briefings were conducted at various SGH departmental meetings to create awareness among department heads and clinicians. It was essential to maintain good communication links at all time to ensure that issues are appropriately managed in a timely manner to minimise risk [7].

From initial planning to completion of the CoPAT programme, the ID speciality/ OPAT nurse played a pivotal liaison role between multiple parties to ensure smooth discharge and service delivery. She was also the point-of-contact between the community nurses and the OPAT/ID physicians during escalation when complication arises. As CoPAT was introduced as the extension of the OPAT, the OPAT/ ID physician was responsible for updating the community nursing team for changes to the treatment plan. In addition to early screening and effective communication among teams, having sufficient nursing manpopwer to support the service on Sundays and Public Holidays were also important in order to sustain the service and support the future expansion.

Since the development of CoPAT, four patients were enrolled for the pilot between September and November 2018 (Table 1). They were on IV Ceftriaxone $2 \mathrm{gm}(\mathrm{n}=3)$ and IV Ertapenem $1 \mathrm{gm}(\mathrm{n}=1)$ once a day dosing. No adverse effects were reported, and there was a total of 30 bed days saved for the hospital and $\$ 16,652$ saved for the patients.

Moving forward, this service has been expanded to include all antibiotics, except Vancomycin because it requires therapeutic level monitoring. Other than the intravenous route, the service will also include patients requiring prolonged antibiotics through central venous catheter. As catheter-related complications can be high as compared to IV cannula, training and careful assessment are essential.

\section{Conclusion}

The patient-centric CoPAT service led by the hospital's OPAT team and community nursing team offers greater comfort (at patient's home), risk reduction and cost savings (shortened hospitalisation stay and reduced associated risk of hospital-acquired infections) to frail patients requiring prolonged intravenous antibiotics therapy. With the promising outcomes, the scope of service will continue to evolve to benefit more patients.

\section{References}

1. Chapman ALN, Seaton RA, Cooper MA, Hedderwick S, Goodall V, et al. (2012) Good practice recommendations for outpatient parenteral antimicrobial therapy (OPAT) in adults in the UK: A consensus statement. J Antimicrob Chemother 67: 1053-1062.

2. Fisher D, Michaels J, Hase R, Zhang J, Kataria S, et al. (2017) Outpatient parenteral antibiotic therapy (OPAT) in Asia: missing an opportunity. J Antimicrob Chemother 72: $1221-1226$.

3. Halilovic J, Christensen CL, Nguyen HH. Managing an outpatient parenteral antibiotic therapy team: challenges and solutions. Ther Clin Risk Manag 10: 459-465.

4. Candel FJ, Julián-Jiménez A, González-Del Castillo J (2016) Current status in outpatient parenteral antimicrobial therapy: a practical view. Revista Espanola de Quimioterapia 29: 55-68.

5. Mitchell ED, Czoski Murray C, Meads D, Minton J, Wright J, et al. (2017) Clinical and cost-effectiveness, safety and acceptability of community intravenous antibiotic service models: CIVAS systematic review. BMJ Open 7: e013560.

6. Rockwood K, Song X, MacKnight C, Bergman H, Hogan DB, et al. (2005) A global clinical measure of fitness and frailty in elderly people. CMAJ 173: 489-495. [Crossref]

7. Davis G, Woodhead J (2016) Setting up a unique outpatient parenteral antimicrobial therapy (OPAT) service. J Comm Nurs 30: 61-65.

Copyright: (C2019 Yi X. This is an open-access article distributed under the terms of the Creative Commons Attribution License, which permits unrestricted use, distribution, and reproduction in any medium, provided the original author and source are credited. 